\title{
Bacterial endocarditis following repeated tattooing
}

\author{
D K Satchithananda, J Walsh, P M Schofield
}

\begin{abstract}
Body decoration in the form of tattooing is becoming increasingly popular, especially among younger age groups. Although serious infections following tattooing are rare they are well documented. The first reported case of endocarditis caused by repeated tattooing in an individual with known valvar heart disease is presented. (Heart 2001;85:11-12)
\end{abstract}

Keywords: endocarditis; tattooing

A 28 year old man with a congenital functionally bicuspid aortic valve was tattooed monthly for a period of five years in a tattoo parlour (fig 1). One week after his final tattooing he developed symptoms of malaise, rigors, and profuse night sweats. He eventually presented to hospital a week later following an embolic event to his left second toe. On examination he had evidence of clinically severe aortic regurgitation and heart failure. Transthoracic echocardiography demonstrated a vegetation on the aortic valve, severe aortic regurgitation, and mildly impaired left ventricular function (fig 2). $\mathrm{He}$ had no preceding history of cellulitis, abscesses, or intravenous drug abuse.

Staphylococcus aureus sensitive to vancomycin, gentamicin, erythromycin, penicillin, and flucloxacillin was isolated from multiple blood cultures. After 48 hours of appropriate antibiotic treatment (vancomycin and gentamicin) an aortic valve replacement was performed using a St Jude's metal bileaflet prosthetic

Papworth Hospital, Papworth Everard, Cambridgeshire CB3 8RE, UK

D K Satchithananda J Walsh

P M Schofield

Correspondence to: Dr Satchithananda dargoi.satchi@excite.co.uk

Accepted 28 September 2000

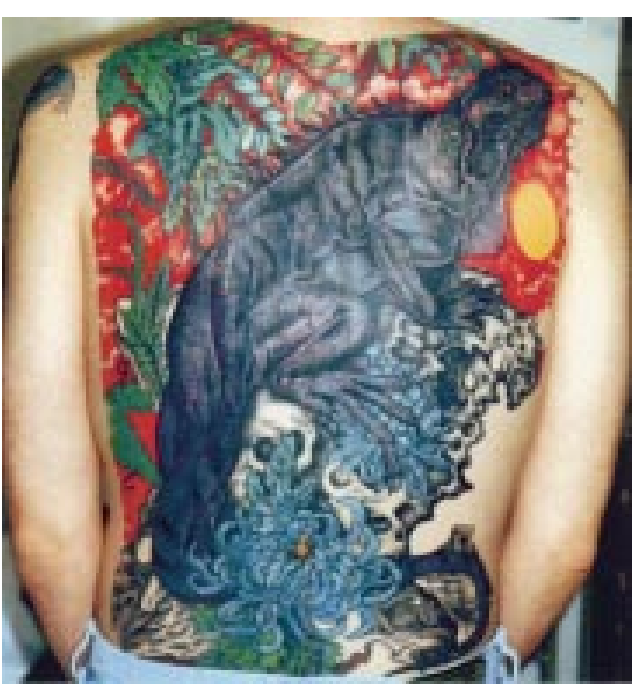

Figure 1 Patient's back with completed tattoo.

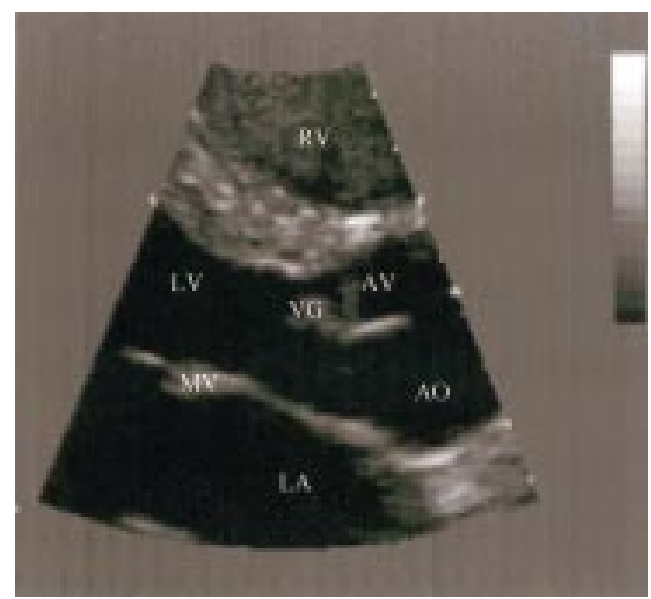

Figure 2 Transthoracic echocardiogram (parasternal long axis) demonstrating the aortic valve vegetation. $R V$, right ventricle; $L V$, left ventricle; $A O$, aorta; $L A$, left atrium; $M V$, mitral valve; $A V$, aortic valve; $V G$, vegetation.

valve. Cultures taken from the patient's explanted native aortic valve grew $S$ aureus of similar sensitivities to that isolated in the initial blood cultures. No aortic valve abscess was seen at the time of surgery.

On the advice of the microbiologist, high dose flucloxacillin was substituted for vancomycin postoperatively. The patient became apyrexial and was clinically well in the initial postoerative period. He had a corresponding reduction in his inflammatory markers. Unfortunately he became pyrexial on the 10th postoperative day. $\mathrm{He}$ also developed a new harsh systolic murmur, a rise in inflammatory markers, and radiographic evidence of heart failure. Transoesophageal echocardiography revealed a prosthetic valve abscess eroding to create a ventricular septal defect (VSD). Surgical correction of the VSD and a redo aortic valve replacement (using a tissue valve) was performed as an emergency procedure. Cultures from the excised prosthetic valve and from the debrided aortic root were sterile. The patient's remaining postoperative course was uncomplicated. He was subsequently discharged after completing a further two weeks of intravenous gentamicin, six weeks of flucloxacillin (four weeks intravenously and two weeks orally), and six weeks of oral rifampacin. $\mathrm{He}$ continues to be well with no features of endocarditis.

\section{Discussion}

Body piercing and tattooing are becoming increasingly fashionable and socially accept- 
able. Tattooing is performed in establishments which vary in their adherence to aseptic techniques. ${ }^{1}$ These establishments use an automated system which drives up to 14 dye impregnated needles into the dermis several hundred times per minute. ${ }^{2}$ The process is repeated over an area of skin corresponding to the chosen design. This leaves an area of superficial bleeding which, once healed, reveals the completed tattoo. Although the incidence of staphylococcal bacteraemia following tattooing is unknown it remains the most likely source of sepsis in our patient. The antibiotic sensitivities demonstrated by the causative $S$ aureus are also suggestive of a community acquired endocarditis. ${ }^{3}$

This is the first reported case of endocarditis related to tattooing in the literature. Other infective complications following tattooing are rare, but well documented. ${ }^{2}$ The absence of other reports of endocarditis caused by tattooing may reflect either higher standards of sterility in tattoo establishments than were previously suggested, ${ }^{1}$ or the lack of recognition of tattooing as a risk factor in staphylococcal endocarditis.

Endocarditis of a bicuspid aortic valve has a high rate of serious infective complications (for example, heart failure $72 \%$, valvar or myocardial abscesses $30 \%$ ), and leads to valve replacement during the initial admission in up to $82 \%$ of cases (often as an emergency procedure). ${ }^{5}$ Many physicians reason that these potentially fatal consequences warrant recommendation of antibiotic prophylaxis in patients with valvar heart disease considering body piercing or tattooing. ${ }^{6}$ There is, however, currently no evidence or guidelines to support this course of action.

Our patient was aware of his valvar heart disease, but perceived tattooing as a "low" risk procedure for endocarditis and therefore failed to seek medical advice before embarking on his body decoration (fig 1). It is important that physicians are fully aware of the potential complications related to tattooing, in order to provide patients with valvar heart disease (and their tattooists) the advice required to make a more informed risk assessment of any intended tattoo.

Dr DK Satchithananda is supported by a research grant from the Garfield Weston Trust. We are grateful to Ms K Byrne for her secretarial services. We are indebted to the patient for allowing us to bring his case to light.

1 Goudey RE, Thomson SC. Evaluation of infection control in registered tattooing premises in Victoria, 1994. Aust NZ in registered tattooing premises

2 Scutt RWB. The medical hazards of tattooing. Br 7 Hosp Med 1972;8:194-202.

3 Waldvogel FA. Staphylococcus aureus. In: Mandell GL, Bennett JE, Dolin R, eds. Principles and practice of infectious diseases. Churchill Livingstone, 2000:2069-92.

4 Long GE, Rickman LS. Infectious complications of tattoos. Clin Infect Dis 1994;18:610-19.

5 Llamas CC, Eykyn SJ. Bicuspid aortic valve-a silent danger: analysis of 50 cases of infective endocarditis. Clin Infect Dis 2000;30:336-41.

6 Cetta F, Graham LC, Lichtenberg RC, et al. Piercing and tattooing in patients with congenital heart disease: patients and physician perspectives. F Adolesc Health 1999;24:160-2.

\section{IMAGES IN CARDIOLOGY}

\section{Angiographic features of endomyocardial fibrosis}

A 32 year old woman had restrictive cardiomyopathy caused by endomyocardial fibrosis proven at biopsy. Left ventricular injection showed grade III obliteration of the cavity (obliteration of half or more of cavity). Apical obliteration was lobulated. Obliteration of the apex resulted in a short axis dimension that was longer than the long axis dimension. There was no mitral regurgitation. The mitral valve crescent was indistinct because of fibrosis of the posterior cusp. There was myocardial calcification in the inferior wall (arrowhead bottom right).

K M KRISHNAMOORTHY

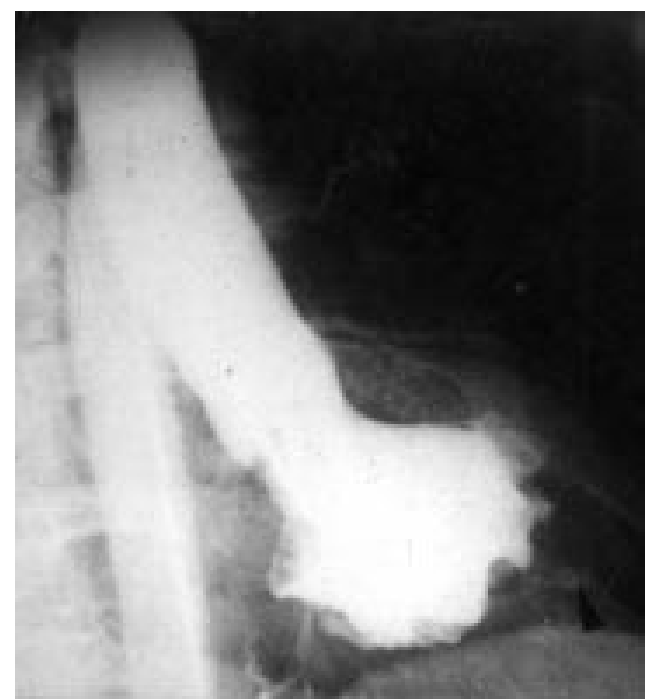

